\title{
REVUE RAPIDE : 381 SUJETS IMPUISSANTS, EXPLORES EN 2 ANS, DONT 207 AVEC PROSTAGLANDINE E1
}

\author{
Michel ETCHEVERRY* - J. TIGNOL **
}

\author{
*Service Uro-Andrologie - Clinique Bordeaux-Nord, 13, Rue Claude Boucher - 33300 BORDEAUX \\ **Université Bordeaux II - Service de Psychiatrie, Centre Carrère - 121, rue de la Béchade - 33076 BORDEAUX CEDEX \\ Groupe d'Etude des Troubles Sexuels Masculins. (G.E.T.S.M.) UNIVERSITE BORDEAUX II
}

BRIEF PRESENTATION OF 381 IMPOTENT PATIENTS INVESTIGATED DURING A TWO-YEAR PERIOD, INCLUDING 207 TREATED WITH PROSTAGLANDIN E1. During the last two years, 381 impotent patients have been tested, 174 with papaverine and 207 with prostaglandin E1 (PGE1 : Alprostadil). More positive responses were obtained using PGE1 (76.8\%) than papaverine $(44.8 \%)$, and some patients whose response to an initial PGE1 test was negative demonstrated a positive effect after a second injection. Two non-responders to PGE1 showed a positive response to papaverine + phentolamine. Tolerance of PGEI was extremely good, provoking fewer prolonged erections than papaverine. Pain following intracavernosal injection of PGEI was distressing in $10 \%$ of cases, all young men with psychogenic impotence. A therapeutic trial of repeated injections, carried out in 49 selected patients with mild organic dysfunction but strong psychogenic inhibition, typically showed a rapid clinical improvement. Moreover, 16 patients with cavernosal sclerosis and vascular impotence who were treated by injections two or three months apart (instead of having a prosthetic implant) showed a recovery of sexual activity. Consequently, during these two years only 35 patients $(9.1 \%)$ were treated surgicarly, 21 by the implantation of hydraulic prostheses. The significant benefits of PGE1, especially the ease of self-administration, demonstrate the need for new therapeutic trials. Key words : Prostaglandin E1, male impotence, treatment. Andrologie, 1991, 1 : 136-137.

Depuis Janvier 1990, 422 sujets ont été reçus pour impuissance. Parmi eux, 381 ont subi un bilan comportant les inventaires usuels de débrouillage, pléthysmographie des érections nocturnes, Doppler-couleur pulsé, cavernosométrie et graphie, examens neurologiques, lorsqu'ils ont été jugés utiles, puis les tests par injections intracaverneuses de drogues vasoactives (D.V.I.C.) :

- 174 tests à la Papavérine (20 à $80 \mathrm{mg}$ )

- 207 tests à la Prostaglandine E1

(PGE1 20 à 50 mcg).

\section{RESULTATS : TOUTES ETIOLOGIES CONFONDUES}

Sur 174 tests à la Papavérine : 78 positifs, soit $44,8 \%$. Trois érections prolongées ont nécessité 3 ponctions, suivies de succès; 15 patients ont appris les auto-injections ; une érection prolongée de 11 heures, après prostatectomie totale élargie, a bien régressé, de même qu'une de 27 heures, chez un psychogène qui n'a pas cru bon de se déranger, résolue spontanément et sans séquelles.

Sur 207 tests à la PG E1 : 159 positifs, soit 76,8 $\%$. Aucune érection prolongée n'a été notée, 12 érections de 4 à 6 heures ont cessé spontanément. Pas de complications locales ou systémiques. Un urticaire géant, avec ædème de QUINCKE est apparu dans les 12 heures, chez un patient pour lequel la coïncidence a été retenue, en l'absence de toute autre étiologie vraisemblable ; 14 patients ont appris les auto-injections (12 impuissants psychogènes et 2 opérés de prostatectomie totale). Excellente impression globale, rapports parfaitement satisfaisants.

Parmi les 48 sujets avec test négatif, 27 sont devenus positifs pour une deuxième injection à dosage plus élevé. Il s'agissait d'impuissances vasculo-caverneuses modérées. Deux fois, des tests négatifs à la PGE1 se sont positivés avec une association Papavérine $80 \mathrm{mg}+$ Phentolamine $10 \mathrm{mg}$, chez des patients associant insuffisance artérielle et fuite veineuse. Les douleurs sont restées, dans notre expérience, d'une modeste incidence. A l'injection, un patient sur deux décrit une brûlure fugitive d'intensité variable. Durant la période d'érection, 16 patients sur $159(10 \%)$ ont ressenti une tension pénible, avec, une fois, des douleurs pelviennes ; 6 ont qualifié la sensation de très désagréable ; chaque fois il s'agissait de patients jeunes, impuissants psychogènes, au test très positif pour une faible dose.
Remarques : Cinq patients antérieurement opérés de fuite veineuse par résection étendue et dont le résultat s'était altéré nettement, ont eu des tests positifs. Neuf fuites veineuses bien caractérisées, non opérées, ont des pharmacotests positifs avec la PGE1 et pas avec la Papavérine. Deux sujets impuissants "invétérés", réunissant plusieurs facteurs intriqués, ont également des tests positifs avec la PGE1 seule.

Un sujet, atteint de maladie de RAYNAUD, a été testé, en pleine crise; elle a cessé immédiatement.

\section{PRISE EN CHARGE DE CERTAINS PATIENTS PAR REINJECTIONS SUCCESSIVES}

En 1990, 14 patients, puis en 1991, 35 autres, ont été soumis à des essais de traitement par des injections itératives espacées de 15 jours, et en ont subi de 2 à 5 . Il s'agit de sujets difficilement classables, présentant des dysérections variables, avec mélange de problèmes organiques vasculocaverneux modérés mais réels, et d'anxiété de performance, bref une forme clinique assez fréquente (49 sujets, soit 12,8\%) dans notre pratique. Ces impuissants-là sont demandeurs de solutions faciles et rapides, surtout après l'échec habituel d'autres approches de leur dysérection. Les résultats paraissent très encourageants, mais difficiles encore à chiffrer. C'est la réassurance, la reprise de confiance en soi qui semble dominer, au spectacle d'érections de bonne qualité, et après quelques rapports mieux réussis.

Enfin, nous traitons $16(4,2 \%)$ autres patients par des injections régulièrement répétées, tous les 2 à 3 mois (PGE1 50 à $75 \mathrm{mcg}$ ). Ce sont des impuissants vasculaires mixtes, avec sclérose caverneuse, mais dont les tests se positivent peu à peu. Ils reprennent des rapports après les injections et l'activité s'estompe à nouveau progressivement. Ce choix thérapeutique est fait en alternative à la pose de prothèses péniennes, car nous pensons que les interventions à visée étiologique ont de faibles chances d'amener des satisfactions. 


\section{COMMENTAIRES}

Après 15 années de pratique des troubles sexuels masculins, vus sous l'angle de la consultation urologique, et avec l'aide de notre entourage psycho-sexologique du G.E.T.S.M., nous dressons un rapide bilan de notre attitude actuelle : 381 sujets explorés avec DVIC, toutes étiologies confondues, $44,8 \%$ de tests positifs avec la Papavérine, $76,8 \%$ avec la PGE1. La tendance est de développer l'utilisation de la PG El, de par son action plus constante, et son innocuité. Nous pouvons ainsi développer une prise en charge différente, avec des essais thérapeutiques nouveaux, en associant la prise en charge psycho-sexologique à la réassurance apportée par les injections itératives.

Nous continuons à rester modérés dans nos indications opératoires: 35 interventions sanglantes en 2 ans sur 381 sujets $(9,1 \%)$ dont 3 revascularisations par la veine dorsale profonde, 11 résections veineuses étendues, 21 implantations de prothèses hydrauliques, ceci chez des impuissants organiques ne répondant pas aux injections de PGEI. L'apprentissage des auto-injections en vue de prothèse chimique a séduit 29 sujets $(7,3 \%)$. Les injections répétées sont réalisées chez 65 patients $(49+16)$, qui sont ainsi soustraits, pour l'instant aux traitements invasifs.

Cet ensemble représente $33,4 \%$ de notre recrutement ; $66 \%$ des consultants pour impuissance sont pris en charge de façon non invasive, par conséquent. La PGEl est pour une part notoire dans cette évolution.

\section{CONCLUSION}

Depuis deux ans, nous utilisons de plus en plus la PGE1 en pratique courante pour explorer et essayer de traiter les impuissants. Notre expérience confirme une réponse plus régulière aux pharmaco-tests, une excellente tolérance, et surtout l'absence de problèmes en auto-injections. Il semble que l'on soit en droit d'isoler une certaine catégorie de patients (17\%) pouvant être améliorés par des injections répétées, lorsque l'atteinte organique vasculo-caverneuse est modérée, en association avec une prise en charge psycho-sexuelle.
RESUME : Au cours des 2 demières années, nous avons testé 381 patients, 179 avec Papavérine, 207 avec la PG E1. Les réponses positives sont plus fréquentes avec la PGE1 ( $76,8 \%$ ) qu'avec la Papavérine $(44,8 \%)$. La tolérance de la PGEl a été bonne ; elle provoque moins d'érections prolongées que la Papavérine. La douleur provoquée par l'IIC de PG El est pénible dans $10 \%$ des cas, mais toujours chez des impuissants psychogènes jeunes. Un essai de traitement par injections itératives a été tenté chez 49 patients choisis pour leur atteinte organique modérée. Les résultats sont intéressants, du fait d'une amélioration souvent rapide. Durant ces deux années, marquées par l'introduction de la PGEI dans notre pratique, nous n'avons opéré que 35 patients, dont 21 prothèses hydrauliques. Nous pensons que les effets intéressants de l'Alprostadil autorisent des essais thérapeutiques nouveaux, et facilitent le développement des auto-injections. Mots clés : Prostaglandine E1, Pharmaco-tests, Traitements intracaverneux. Andrologie, 1991, 1 : 136-137. 